\title{
APPROXIMATION AND ENTROPY NUMBERS OF COMPACT SOBOLEV EMBEDDINGS
}

\author{
LESZEK SKRZYPCZAK \\ Faculty of Mathematics and Computer Science, A. Mickiewicz University \\ Umultowska 87, 61-614 Poznań, Poland \\ E-mail: lskrzyp@amu.edu.pl
}

Dedicated to Zbigniew Ciesielski on the occasion of his 70th birthday

\begin{abstract}
The aim of the paper is twofold. First we give a survey of some recent results concerning the asymptotic behavior of the entropy and approximation numbers of compact Sobolev embeddings. Second we prove new estimates of approximation numbers of embeddings of weighted Besov spaces in the so called limiting case.
\end{abstract}

The idea of entropy numbers goes back to the works of L. S. Pontryagin and L. G. Schnirelmann and of A. N. Kolmogorov in the 1930s on the metric entropy of compact sets in metric spaces. The definition of approximation numbers has its roots in D. Eh. Allakhverdiev's paper published in 1957. It is proved there that singular numbers of a compact operator acting in a Hilbert space coincide with a quantities that nowadays are called approximation numbers. The abstract Banach space setting of the topic was given by A. Pietsch who developed the theory of operator ideals and s-numbers. Very important contribution to the subject was provided in 1980 by B. Carl's observation that entropy numbers of a compact operator acting in a Banach space are related to its eigenvalues by a simple inequality.

Embeddings between function spaces from the point of view of entropy numbers were first investigated by A. N. Kolmogorov and V. M. Tikhomirov in 1959. They found the asymptotic behavior of the natural embedding of $C^{k}\left([0,1]^{n}\right)$ into $C\left([0,1]^{n}\right)$. Entropy numbers of embeddings of Sobolev spaces were first treated by M. S. Birman and M. Z. Solomyak in 1967. The great impetus to the development of the study of asymptotic behavior of the entropy and approximation numbers of embedding between function spaces was given by D. E. Edmunds and H. Triebel in the 80s and 90s of the last century. They proved several sharp estimates, gave the quasi-Banach version of the theory and

2000 Mathematics Subject Classification: 46E35, 41A46.

The paper is in final form and no version of it will be published elsewhere. 
moreover developed a method of application of the estimates to spectral properties of some elliptic pseudo-differential operators.

Recently it has become obvious that the simplest and quite efficient way of studying the asymptotic behavior of both entropy and approximation numbers leads through the discretization of the function spaces. This strategy was used in a series of papers by Th. Kühn, H. G. Leopold, W. Sickel and the author [27]-[31], but also in D. Haroske, H. Triebel [21] and B. Tomasz and the author [44], cf. also [43]. The first step of the method is to reduce the problem from the function spaces level to related sequence spaces. Then one deals with estimates for embeddings of the sequence spaces, using or developing the knowledge about corresponding operator ideals and diagonal operators. Theorems stated in Section 3 can be proved using this method. The strategy is presented in the last section in which we prove one of the theorems of Section 3.

1. Compact Sobolev embeddings. Sobolev embeddings we mention above are the embeddings between function spaces of Besov and Lizorkin-Triebel type. In the paper we concentrate on (inhomogeneous) Besov spaces. But all theorems stated in Section 3 except for Theorem 4 and Theorem 5 hold also for Sobolev and more general Lizorkin-Triebel spaces.

We assume that the reader is acquainted with the definition and basic properties of Besov spaces $B_{p, q}^{s}\left(\mathbb{R}^{d}\right), 0<p, q \leq \infty$ and $s \in \mathbb{R}$. Triebel's books [49] and [51] are the classical references here but one can consult also [14] and many other books. In this section we recall a definition and a few properties of weighted function spaces. For simplicity we restrict our attention to the Besov spaces with indices $p$ and $q$ greater than or equal to one. In that case the spaces $B_{p, q}^{s}\left(\mathbb{R}^{d}\right)$ are Banach spaces. If $p<1$ or $q<1$ then the corresponding spaces are quasi-Banach. Most of the results we present in the paper have their quasi-Banach counterparts. We will denote the continuous embeddings between Banach spaces by $\hookrightarrow$.

Let $p_{0} \leq p_{1}$ and $s_{0} \geq s_{1}$. It is well known that if $\delta=s_{0}-s_{1}-d\left(\frac{1}{p_{0}}-\frac{1}{p_{1}}\right)>0$ or $\delta=0$ and $q_{0} \leq q_{1}$ then

$$
B_{p_{0}, q_{0}}^{s_{0}}\left(\mathbb{R}^{d}\right) \hookrightarrow B_{p_{1}, q_{1}}^{s_{1}}\left(\mathbb{R}^{d}\right) .
$$

The above embeddings are never compact. To get compact embeddings we must take a smaller source space or a bigger target space. We will focus our attention on the following three situations that found different applications in analysis:

(i) Spaces on bounded domains. Let $\Omega$ be a bounded domain in $\mathbb{R}^{d}$ with smooth or Lipschitz boundary. We define the Besov spaces on $\Omega$ by restriction, i.e.

$$
\begin{aligned}
B_{p, q}^{s}(\Omega) & =\left\{f \in \mathcal{D}^{\prime}(\Omega): f=\left.g\right|_{\Omega}, \text { for some } g \in B_{p, q}^{s}\left(\mathbb{R}^{d}\right)\right\}, \\
\left\|f \mid B_{p, q}^{s}(\Omega)\right\| & =\inf \left\|g \mid B_{p, q}^{s}\left(\mathbb{R}^{d}\right)\right\|,
\end{aligned}
$$

where the infimum is taken over all possible $g \in B_{p, q}^{s}\left(\mathbb{R}^{d}\right)$.

Given any $a \in \mathbb{R}$ we shall write $a_{+}=\max (a, 0)$. The embedding

$$
B_{p_{0}, q_{0}}^{s_{0}}(\Omega) \hookrightarrow B_{p_{1}, q_{1}}^{s_{1}}(\Omega)
$$

is compact if and only if $s_{0}>s_{1}$ and $\delta_{+}=s_{0}-s_{1}-d\left(\frac{1}{p_{0}}-\frac{1}{p_{1}}\right)_{+}>0$. 
(ii) Spaces of radial functions and distributions. We recall that the distribution $f$ is called radial if $f(\varphi)=f(\varphi \circ R)$ for any test function $\varphi$ and any rotation $R$ around the origin. We put

$$
R B_{p, q}^{s}\left(\mathbb{R}^{d}\right)=\left\{f \in B_{p, q}^{s}\left(\mathbb{R}^{d}\right): f \text { radial }\right\}
$$

The embedding

$$
R B_{p_{0}, q_{0}}^{s_{0}}\left(\mathbb{R}^{d}\right) \hookrightarrow R B_{p_{1}, q_{1}}^{s_{1}}\left(\mathbb{R}^{d}\right)
$$

is compact if and only if $1 \leq p_{0}<p_{1} \leq \infty$ and $\delta:=s_{0}-s_{1}-d\left(\frac{1}{p_{0}}-\frac{1}{p_{1}}\right)>0$.

(iii) Weighted function spaces. We restrict here to the function spaces with polynomial weights

$$
w_{\alpha}(x)=\left(1+|x|^{2}\right)^{\alpha / 2}, \quad \alpha>0 .
$$

The weighted Besov spaces with the above weight can be defined in the following way:

$$
B_{p, q}^{s}\left(\mathbb{R}^{d}, w_{\alpha}\right)=\left\{f \in \mathcal{S}^{\prime}\left(\mathbb{R}^{d}\right):\left\|f\left|B_{p, q}^{s}\left(\mathbb{R}^{d}, w\right)\|=\| f w\right| B_{p, q}^{s}\left(\mathbb{R}^{d}\right)\right\|<\infty\right\} .
$$

The study of the properties of embeddings between two weighted spaces can be easily reduced to properties of embedding of weighted spaces into unweighted spaces by taking a new space with weight equal to the ratio of the two original weights. The embedding

$$
B_{p_{0}, q_{0}}^{s_{0}}\left(\mathbb{R}^{d}, w_{\alpha}\right) \hookrightarrow B_{p_{1}, q_{1}}^{s_{1}}\left(\mathbb{R}^{d}\right)
$$

is compact if and only if $\min (\alpha, \delta)>d\left(\frac{1}{p_{1}}-\frac{1}{p_{0}}\right)_{+}$, where $\delta=s_{0}-s_{1}-d\left(\frac{1}{p_{0}}-\frac{1}{p_{1}}\right)$.

So called limiting embeddings are other interesting cases. They are embeddings into spaces that do not belong to the Besov or Triebel-Lizorkin scales. In particular embeddings of the Trudinger-Strichartz type are embeddings into exponential Orlicz spaces $E_{\nu, \rho}\left(\mathbb{R}^{d}\right)$. The latter are Orlicz spaces generated by Orlicz functions $t \rightarrow t^{\nu} \exp \left(t^{\rho}\right)$ (one can use also $t \rightarrow \exp \left(t^{\rho}\right)-1$ on bounded domains). It is well known that the space $B_{p, q}^{d / p}\left(\mathbb{R}^{d}\right)$ is not contained in $L_{\infty}\left(\mathbb{R}^{d}\right)$ but we have $B_{p, q}^{d / p}\left(\mathbb{R}^{d}\right) \hookrightarrow E_{\nu, \rho}\left(\mathbb{R}^{d}\right)$ if $\nu \geq p$ and $\rho \leq q^{\prime}=\frac{q}{q-1}, 1<q<\infty, 1<p<\infty$. This justifies the name "limiting embeddings". In a similar way a function $f$ belonging to the (fractional) Sobolev space $H_{p}^{1+d / p}\left(\mathbb{R}^{d}\right)$, $1<p<\infty$, is Hölder-continuous with exponent $\alpha<1$ but not Lipschitz-continuous. It was noticed by Brézis and Wainger that $f$ is "almost" Lipschitz-continuous in the sense that

$$
|f(x)-f(y)| \leq C|x-y||\log | x-y||^{1 / p^{\prime}}\left\|f \mid H_{p}^{1+d / p}\left(\mathbb{R}^{d}\right)\right\|
$$

with $0<|x-y|<\frac{1}{2}, x, y \in \mathbb{R}^{d}$. In the paper we restrict our attention to the embeddings of the Besov spaces into spaces of the same type.

REMARK 1. (a) The compactness of the embeddings of classical Sobolev spaces defined on bounded domains into $L_{p}$ spaces was noticed by V. I. Kondrashov in 1945.

(b) The fact that symmetry as well as weights can be used to generate compactness of embeddings on $\mathbb{R}^{d}$ is known since the seventies, cf. e.g. Strauss [46], Coleman, Glazer and Martin [10] and Lions [32] about the first order Sobolev spaces.

(c) It follows immediately from the definition that an operator $f \rightarrow w f$ is an isomorphic mapping from $B_{p, q}^{s}\left(\mathbb{R}^{d}, w\right)$ onto $B_{p, q}^{s}\left(\mathbb{R}^{d}\right)$. There are different ways to introduce weighted 
function spaces, but for the polynomial weights we use, these different approaches coincide, cf. [33, 40, 41, 14].

(d) The Trudinger-Strichartz embeddings were first considered by Trudinger [53] and Strichartz [47], whereas the Brézis-Wainger embeddings were introduced in [4]. There is a quite large literature on entropy and approximation numbers of these embeddings, cf. e.g. [48, 26, 42] about the Trudinger-Strichartz embeddings and $[11,9]$ about the Brézis-Wainger embeddings.

2. Approximation and entropy numbers. Approximation numbers and entropy numbers are popular tools for qualitative description of the compactness of a bounded linear operator with applications to the spectral theory. Approximation numbers measure the closeness by which a bounded operator may be approximated by linear maps of finite range, whereas entropy numbers measure compactness of the operator by means of finite coverings of images of the unit ball.

Let us briefly recall the definition and basic properties of approximation and entropy numbers. Let $A_{0}$ and $A_{1}$ be two complex Banach spaces and let $T: A_{0} \rightarrow A_{1}$ be a bounded linear operator.

DeFinition 1 . The $k$ th approximation number $a_{k}(T)$ of a bounded linear operator $T$ : $A_{0} \rightarrow A_{1}$ is the infimum of all numbers $\|T-F\|$ where $F$ runs over all continuous linear operators $F: A_{0} \rightarrow A_{1}$ of rank smaller than $k$.

The approximation numbers $a_{k}(T)$ form a decreasing sequence with $a_{1}(T)=\|T\|$. If the sequence converges to zero then the operator $T$ is compact. The opposite implication in general is not true. It may happen that $\lim _{k \rightarrow \infty} a_{k}(T)>0$ for some compact $T$ if $A_{1}$ fails to have the approximation property. The approximation numbers have in particular the following properties:

- (additivity) $a_{n+k-1}\left(T_{1}+T_{2}\right) \leq a_{k}\left(T_{1}\right)+a_{n}\left(T_{2}\right)$,

- (multiplicativity) $a_{n+k-1}\left(T_{1} T_{2}\right) \leq a_{k}\left(T_{1}\right) a_{n}\left(T_{2}\right)$,

- (rank property) $a_{k}(T)=0 \Rightarrow \operatorname{rank}(T)<k$.

Definition 2. Let $A_{0}, A_{1}$ be two complex Banach spaces and let $T$ be a linear and continuous operator from $A_{0}$ into $A_{1}$. Let $k \in \mathbb{N}$. The $k$-th entropy number $e_{k}\left(T: A_{0} \rightarrow\right.$ $\left.A_{1}\right)$ is the infimum of all numbers $\varepsilon>0$ such that there exist $2^{k-1}$ balls in $A_{1}$ of radius $\varepsilon$ which cover the image of the unit ball $U:=\left\{x \in X:\|x\|_{A_{0}} \leq 1\right\}$ under the mapping $T$.

Entropy numbers have properties similar to approximation numbers: they form a decreasing sequence with $e_{1}(T)=\|T\|$; they have additivity and multiplicativity properties, but not the rank property. Moreover,

$$
T \text { is compact } \Leftrightarrow \lim _{k \rightarrow \infty} e_{k}\left(T: A_{0} \rightarrow A_{1}\right)=0 .
$$

For details proofs and other properties we refer to the monographs [7, 14, 25, 37].

We shall use the operator ideals $\mathcal{L}_{p, \infty}^{(a)}$ and $\mathcal{L}_{p, \infty}^{(e)}, 0<p<\infty$, formed by all bounded linear operators $T$ between Banach spaces such that the quasi-norms

$$
L_{p, \infty}^{(a)}(T)=\sup _{k} k^{1 / p} a_{k}(T) \quad \text { and } \quad L_{p, \infty}^{(e)}(T)=\sup _{k} k^{1 / p} e_{k}(T)
$$


respectively, are finite. The classes $\left(\mathcal{L}_{p, \infty}^{(a)}, L_{p, \infty}^{(a)}\right)$ and $\left(\mathcal{L}_{p, \infty}^{(e)}, L_{p, \infty}^{(e)}\right)$ are quasi-normed operator ideals in the sense of A. Pietsch [37, Definition 6.1.1].

Both concepts, entropy and approximation numbers, are related to each other. Namely for a compact operator $T$ there is some constant $c>0$ such that for all $k \in \mathbb{N}$ the inequality $e_{k}(T) \leq c a_{k}(T)$ holds assuming that there is some $c^{\prime}>0$ such that $a_{2^{j-1}}(T) \leq$ $c a_{2^{j}}(T)$ for all $j \in N$, cf. [50].

Both quantities have their importance for spectral theory. For example the Carl inequality links entropy numbers and eigenvalues of the compact operator. More precisely, if $T: A_{0} \rightarrow A_{0}$ is a compact operator and $\left(\lambda_{n}(T)\right)_{n=1}^{\infty}$ is the sequence of all non-zero eigenvalues of $T$, repeated according to algebraic multiplicity and ordered in the decreasing way then

$$
\left|\lambda_{n}(T)\right| \leq \sqrt{2} e_{n}(T),
$$

cf. [6], [8] or monographs [7] and [14].

On the other hand if $T$ is a compact operator acting in the Hilbert space $H$ and $|T|$ is a square root of $T^{*} T$ then the $k$-th approximation number of $T$ coincides with the $k$-th non-zero eigenvalue of $|T|$. In particular if $T$ is nonnegative and self-adjoint then $a_{k}(T)=\lambda_{k}(T)$. Moreover, for any compact operator in $T$ acting in the Hilbert space $H$ we have the following well known Weyl inequality

$$
\prod_{j=1}^{n}\left|\lambda_{j}(T)\right| \leq \prod_{j=1}^{n} a_{j}(T)
$$

For Banach spaces H. König proved the following weaker version of the last inequality:

$$
\left(\sum_{j=1}^{n}\left|\lambda_{j}(T)\right|^{p}\right)^{1 / p} \leq K_{p}\left(\sum_{j=1}^{n} a_{j}^{p}(T)\right)^{1 / p}
$$

where $0<p<\infty$ and $K_{p}$ is an explicit numerical constant depending on $p$, cf. [25].

In [14] D.E. Edmunds and H. Triebel systematically developed a method of investigation of spectral properties of some compact elliptic differential and pseudo-differential operators, both on bounded domains and on $\mathbb{R}^{d}$, cf. also D. Haroske, H. Triebel [20]. One of crucial ingredients of the method is a factorization through compact Sobolev embeddings in order to use estimates of entropy and approximation numbers as well as their relations with spectral theory. Then using the Birman-Schwinger principle they studied the "negative" spectrum of the Schrödinger type operators $H=A-V$, where $A$ is a positive, self adjoint operator in $L_{2}$ and $V$ is a positive potential. One should also mention forerunners by M. S. Birman, M. Z. Solomyak [3], K. Mynbaev and M. Otel'baev [35] and others.

3. Approximation and entropy numbers of Sobolev embeddings. We start with function spaces defined on bounded domains $\Omega \subset \mathbb{R}^{d}$. For convenience we will use the following abbreviations. Let $1 \leq p_{0} \leq p_{1} \leq \infty$ then

$$
\frac{1}{p}=\frac{1}{p_{0}}-\frac{1}{p_{1}} \quad \text { and } \quad \frac{1}{t}=\frac{1}{\min \left(p_{0}^{\prime}, p_{1}\right)}, \quad \frac{1}{p_{0}^{\prime}}=1-\frac{1}{p_{0}} .
$$


Moreover we denote $a_{k} \sim b_{k}$ if there exists a constant $c>0$ independent of $k$ such that

$$
c^{-1} b_{k} \leq a_{k} \leq c b_{k}, \quad k=1,2,3 \ldots
$$

TheOREM 1. Let $\Omega \subset \mathbb{R}^{d}$ be a bounded domain with smooth or Lipschitz boundary. Let $-\infty<s_{1}<s_{0}<\infty$ and $1 \leq p_{0}, p_{1}, q_{0}, q_{1} \leq \infty,\left(p_{0}, p_{1}\right) \neq(1, \infty)$, and suppose that

$$
\delta_{+}=s_{0}-s_{1}-d\left(\frac{1}{p_{0}}-\frac{1}{p_{1}}\right)_{+}>0 .
$$

Then

$$
e_{k}\left(B_{p_{0}, q_{0}}^{s_{0}}(\Omega) \hookrightarrow B_{p_{1}, q_{1}}^{s_{1}}(\Omega)\right) \sim k^{-\frac{s_{0}-s_{1}}{d}}
$$

and

$$
a_{k}\left(B_{p_{0}, q_{0}}^{s_{0}}(\Omega) \hookrightarrow B_{p_{1}, q_{1}}^{s_{1}}(\Omega)\right) \sim k^{-\varkappa}
$$

where

$$
\varkappa= \begin{cases}\frac{\delta_{+}}{d} & \text { if } 1 \leq p_{0} \leq p_{1} \leq 2 \text { or } 2 \leq p_{0} \leq p_{1} \leq \infty, \\ & \text { or } 1 \leq p_{1} \leq p_{0} \leq \infty, \\ \frac{\delta}{d}+\frac{1}{2}-\frac{1}{t} & \text { if } 1 \leq p_{0}<2<p_{1} \leq \infty \text { and } \delta>\frac{d}{t}, \\ \frac{\delta}{d} \cdot \frac{t}{2} & \text { if } 1 \leq p_{0}<2<p_{1} \leq \infty \text { and } \delta \leq \frac{d}{t}\end{cases}
$$

REMARK 2. (a) The estimate (1) was proved by D. E. Edmunds and H. Triebel, cf. $[12,13,14]$. They also proved the estimate (2) except for some cases if $1 \leq p_{0}<2<$ $p_{1} \leq \infty$. These remaining estimates were improved by A. Caetano [5] except for one case, when $\delta_{+}=d / \min \left(p_{0}^{\prime}, p_{1}\right)$. The last step was recently done in [43].

(b) It follows from (1) and (2) that the asymptotic behavior of the entropy numbers and approximation numbers of Sobolev embeddings on bounded domain depends mainly on the difference of the smoothnesses $s_{0}-s_{1}$. For approximation numbers we have an additional effect when we cross the Hilbert case $p=2$.

In the next theorem we will see that the asymptotic behavior of both entropy and approximation numbers is quite different for spaces of radial functions.

Theorem 2. Let $-\infty<s_{1}<s_{0}<\infty, 1 \leq p_{0}<p_{1} \leq \infty$ and $1 \leq q_{0}, q_{1} \leq \infty$ and suppose that

$$
\delta=s_{0}-s_{1}-d\left(\frac{1}{p_{0}}-\frac{1}{p_{1}}\right)>0 .
$$

Then

$$
e_{k}\left(R B_{p_{0}, q_{0}}^{s_{0}}\left(\mathbb{R}^{d}\right) \hookrightarrow R B_{p_{1}, q_{1}}^{s_{1}}\left(\mathbb{R}^{d}\right)\right) \sim k^{-d\left(\frac{1}{p_{0}}-\frac{1}{p_{1}}\right)}
$$

and

$$
a_{k}\left(R B_{p_{0}, q_{0}}^{s_{0}}\left(\mathbb{R}^{d}\right) \hookrightarrow R B_{p_{1}, q_{1}}^{s_{1}}\left(\mathbb{R}^{d}\right)\right) \sim k^{-\varkappa}
$$

where

$$
\varkappa= \begin{cases}\frac{d-1}{p} & \text { if } 1 \leq p_{0}<p_{1} \leq 2 \text { or } 2<p_{0} \leq p_{1} \leq \infty, \\ \frac{d-1}{p}+\frac{1}{2}-\frac{1}{t} & \text { if } 1 \leq p_{0}<2<p_{1} \leq \infty \text { and } \frac{d-1}{p}>\frac{1}{t} \\ \frac{d-1}{p} \cdot \frac{t}{2} & \text { if } 1 \leq p_{0}<2<p_{1} \leq \infty \text { and } \frac{d-1}{p} \leq \frac{1}{t}\end{cases}
$$


Remark 3. (a) The estimate (3) was proved by Th. Kühn, H.-G. Leopold, W. Sickel and the author, cf. [27], whereas (4) was proved by B. Tomasz and the author [44].

(b) In contrast to the last theorem now neither estimates for entropy numbers nor for approximation numbers depend on the difference of smoothnesses $s_{0}-s_{1}$. This means that behavior of radial functions at infinity is dominating. But for approximation numbers we have a similar additional effect when we cross the Hilbert case $p=2$.

(c) The above estimates for spaces of radial functions depend of the geometry of the underlying space. One can consider also the Besov and Sobolev spaces on Riemannian manifolds with bounded geometry e.g. on hyperbolic spaces $\mathbb{H}^{d}$ (the space $\mathbb{H}^{2}$ is the Lobachevsky plane). Let us fix one point $o \in \mathbb{H}^{d}$ and call it the origin. One can regard radial functions on $\mathbb{H}^{d}$ to be functions whose value at a point $x$ depends only on the distance of $x$ to the origin $o$. The notation can be extended to distributions in the standard way. Let $R B_{p, q}^{s}\left(\mathbb{H}^{d}\right)$ denote a Besov space consisting of radial distributions defined on $\mathbb{H}^{d}$. If $-\infty<s_{1}<s_{0}<\infty, 1 \leq p_{0}<p_{1} \leq \infty, 1 \leq q_{0}, q_{1} \leq \infty$ and $\delta=s_{0}-s_{1}-d\left(\frac{1}{p_{0}}-\frac{1}{p_{1}}\right)>0$. then

$$
e_{k}\left(R B_{p_{0}, q_{0}}^{s_{0}}\left(\mathbb{H}^{d}\right) \hookrightarrow R B_{p_{1}, q_{1}}^{s_{1}}\left(\mathbb{H}^{d}\right)\right) \sim k^{-\left(s_{0}-s_{1}\right)} .
$$

This reminds more the behavior of the Sobolev embeddings on bounded domains on $\mathbb{R}^{d}$ than the embeddings of radial spaces on $\mathbb{R}^{d}$, cf. [45].

Now we consider the embeddings of weighted function spaces. As it was mentioned before we restrict our attention to the polynomial weights. First we formulate the result in so called non-limiting case, i.e. when the power of the weight is not equal to the quantity $\delta=s_{0}-s_{1}-d\left(\frac{1}{p_{0}}-\frac{1}{p_{1}}\right)$.

TheOrEm 3. Let $1 \leq p_{0}, p_{1} \leq \infty, 1 \leq q_{0}, q_{1} \leq \infty$ and $-\infty<s_{1}<s_{0}<\infty$. Let $\alpha>0$ and $\frac{1}{\tilde{p}}=\frac{\alpha}{d}+\frac{1}{p_{0}}$. We assume that

(a) $1 \leq p_{0} \leq p_{1} \leq \infty$ or $\tilde{p}<p_{1}<p_{0} \leq \infty$,

(b) $\alpha \neq s_{0}-s_{1}-d\left(\frac{1}{p_{0}}-\frac{1}{p_{1}}\right)=: \delta$ and $\delta>0$. Then

$$
e_{k}\left(B_{p_{0}, q_{0}}^{s_{0}}\left(\mathbb{R}^{d}, w_{\alpha}\right) \hookrightarrow B_{p_{1}, q_{1}}^{s_{1}}\left(\mathbb{R}^{d}\right)\right) \sim k^{-\varkappa},
$$

where

$$
\varkappa= \begin{cases}\frac{s_{0}-s_{1}}{d} & \text { if } \frac{d}{\widetilde{p}}<\delta<\alpha, \\ \frac{\alpha}{d}+\frac{1}{p_{0}}-\frac{1}{p_{1}} & \text { if } \frac{d}{\widetilde{p}}<\alpha<\delta .\end{cases}
$$

If in addition $\left(p_{0}, p_{1}\right) \neq(1, \infty)$ then

$$
a_{k}\left(B_{p_{0}, q_{0}}^{s_{0}}\left(\mathbb{R}^{d}, w_{\alpha}\right) \hookrightarrow B_{p_{1}, q_{1}}^{s_{1}}\left(\mathbb{R}^{d}\right)\right) \sim k^{-\varkappa}
$$

where

$$
\varkappa= \begin{cases}\frac{\min (\alpha, \delta)}{d} & \text { if } 1 \leq p_{0} \leq p_{1} \leq 2 \text { or } 2 \leq p_{0} \leq p_{1} \leq \infty, \\ \frac{\min (\alpha, \delta)}{d}+\frac{1}{p_{0}}-\frac{1}{p_{1}} & \text { if } \tilde{p} \leq p_{1}<p_{0} \leq \infty, \\ \frac{\min (\alpha, \delta)}{d}+\frac{1}{2}-\frac{1}{t} & \text { if } 1 \leq p_{0}<2<p_{1} \leq \infty \text { and } \min (\alpha, \delta)>\frac{d}{t}, \\ \frac{\min (\alpha, \delta)}{d} \cdot \frac{t}{2} & \text { if } 1 \leq p_{0}<2<p_{1} \leq \infty \text { and } \min (\alpha, \delta) \leq \frac{d}{t} .\end{cases}
$$

REMARK 4. (a) The first results concerning entropy and approximation numbers of embeddings of weighted spaces were proved by D. Haroske and H. Triebel, cf. [19, 20] for 
entropy numbers and D. Haroske [16] for approximation numbers. The above theorem was partly proved there. The final estimates for entropy numbers were proved in [21] and [28]. The related final result concerning approximation number can be found in [43]. (b) The interpretation of the last theorem is the following: if the weight is increasing fast enough near infinity then we are back in the compact case, that means, the asymptotic behavior of the corresponding entropy and approximation numbers coincides with that of the unweighted Besov spaces defined on a bounded domain with smooth or Lipschitz boundary. If the weight is not increasing fast enough, i.e. $\alpha<\delta$, then there is a direct influence of the weight on the asymptotic behavior of the entropy and approximation numbers.

(c) One can also consider more general weights in this context. Entropy numbers for perturbed polynomial weights are considered in [30] and for subpolynomial weights in [31]. Some partial results concerning logarithmic weights can be found also in $[17,18]$.

The estimates in the limiting case are harder to prove and still interesting. For entropy numbers the following theorem was proved in [30].

THEOREM 4. Suppose

$\alpha=\left(s_{0}-\frac{d}{p_{0}}\right)-\left(s_{1}-\frac{d}{p_{1}}\right)>d\left(\frac{1}{p_{1}}-\frac{1}{p_{0}}\right)_{+} \quad$ and set $\tau:=\frac{s_{0}-s_{1}}{d}+\frac{1}{q_{1}}-\frac{1}{q_{0}}$.

(i) If $\tau>0$, then

$$
e_{k}\left(B_{p_{0}, q_{0}}^{s_{0}}\left(\mathbb{R}^{d}, w_{\alpha}\right) \hookrightarrow B_{p_{1}, q_{1}}^{s_{1}}\left(\mathbb{R}^{d}\right)\right) \sim k^{-\frac{s_{0}-s_{1}}{d}}(1+\log k)^{\tau} .
$$

(ii) If $\tau<0$, then

$$
e_{k}\left(B_{p_{0}, q_{0}}^{s_{0}}\left(\mathbb{R}^{d}, w_{\alpha}\right) \hookrightarrow B_{p_{1}, q_{1}}^{s_{1}}\left(\mathbb{R}^{d}\right)\right) \sim k^{-\frac{s_{0}-s_{1}}{d}} .
$$

REMARK 5. (a) Now the asymptotic behavior of the entropy numbers depends on the fine indices of the involved Besov spaces, i.e. on $q_{0}$ and $q_{1}$, a phenomenon that does not occur in non-limiting cases. This was observed first by D. Haroske [17]. She proved part (i) of Theorem 4 under additional restrictions, see [17, 18].

(b) In contrast to the first three theorems the last theorem cannot be immediately extended to Triebel-Lizorkin or even Sobolev spaces. Some partial results related to Sobolev spaces of fractional order can be found in [21].

Much less is known about the asymptotic behavior of the approximation numbers in the limiting case. To the best of our knowledge the following theorem is the first one presenting a partial but sharp result in this direction.

ThEOREM 5. Suppose

$$
\alpha=\left(s_{0}-\frac{d}{p_{0}}\right)-\left(s_{1}-\frac{d}{p_{1}}\right)>d\left(\frac{1}{p_{1}}-\frac{1}{p_{0}}\right)_{+}
$$

and $q_{0}=p_{0}, q_{1}=p_{1},\left(p_{0}, p_{1}\right) \neq(1, \infty)$. Then

$$
a_{k}\left(B_{p_{0}, p_{0}}^{s_{0}}\left(\mathbb{R}^{d}, w_{\alpha}\right) \hookrightarrow B_{p_{1}, p_{1}}^{s_{1}}\left(\mathbb{R}^{d}\right)\right) \sim k^{-\varkappa}(1+\log k)^{\frac{\alpha}{d}},
$$


where

$$
\varkappa= \begin{cases}\frac{\alpha}{d} & \text { if } 1 \leq p_{0} \leq p_{1} \leq 2 \text { or } 2 \leq p_{0} \leq p_{1} \leq \infty, \\ \frac{\alpha}{d}+\frac{1}{p_{0}}-\frac{1}{p_{1}} & \text { if } \tilde{p} \leq p_{1}<p_{0} \leq \infty \\ \frac{\alpha}{d}+\frac{1}{2}-\frac{1}{t} & \text { if } 1 \leq p_{0}<2<p_{1} \leq \infty \text { and } \alpha>\frac{d}{t}, \\ \frac{\alpha}{d} \cdot \frac{t}{2} & \text { if } 1 \leq p_{0}<2<p_{1} \leq \infty \text { and } \alpha \leq \frac{d}{t} .\end{cases}
$$

We give a proof of the above theorem in the next section.

REMARK 6 . The only former sharp result (concerning the above described limiting situation $\delta=\alpha$ ) is that of Mynbaev and Otel'baev [35, $\S 3$, Theorem 9]. They proved that

$$
a_{k}\left(H_{p_{0}}^{s_{0}}\left(\mathbb{R}^{d}, w_{\alpha}\right) \hookrightarrow L_{p_{1}}\left(\mathbb{R}^{d}\right)\right) \sim\left(k^{-1}(1+\log k)\right)^{\frac{\alpha}{d}},
$$

if $s_{0}>0$ and $1<p_{0}<p_{1} \leq 2$ or $2 \leq p_{0}<p_{1}<\infty$. Some, but not sharp, results can be found in [18].

\section{Proof of Theorem 5}

4.1. Discretization of function spaces. We use wavelet bases as a method of discretization. They are now a well developed concept in Besov spaces. In the case of unweighted spaces we refer to the monographs of Y. Meyer [34], P. Wojtaszczyk [54] and the article of G. Bourdaud [2]. Here we are interested in wavelet bases in weighted spaces. We quote the wavelet characterization of weighted Besov spaces proved in [29], but cf. also [39] where the more general weights are considered and [21] where quasi-Banach case is included.

First of all we need to fix some notations. By $\mathbb{N}$ we denote the set of natural numbers, by $\mathbb{N}_{0}$ the set $\mathbb{N} \cup\{0\}$, and by $\mathbb{Z}^{d}$ the set of all lattice points in $\mathbb{R}^{d}$ having integer components. Let $\widetilde{\phi}$ be an orthogonal scaling function on $\mathbb{R}$ with compact support and of sufficiently high regularity. Let $\widetilde{\psi}$ be a corresponding wavelet. Then the tensor product yields a scaling function $\phi$ and associated wavelets $\psi_{1}, \ldots, \psi_{2^{d}-1}$, all defined now on $\mathbb{R}^{d}$. We suppose

$$
\widetilde{\phi} \in C^{N_{1}} \quad \text { and } \quad \operatorname{supp} \widetilde{\phi} \subset\left[-N_{2}, N_{2}\right]
$$

for certain natural numbers $N_{1}$ and $N_{2}$. This implies

$$
\phi, \psi_{i} \in C^{N_{1}} \quad \text { and } \quad \operatorname{supp} \phi, \operatorname{supp} \psi_{i} \subset\left[-N_{3}, N_{3}\right]^{d}, \quad i=1, \ldots, 2^{d}-1 .
$$

We shall use the standard abbreviations

$$
\phi_{j, \ell}(x)=2^{j d / 2} \phi\left(2^{j} x-\ell\right) \quad \text { and } \quad \psi_{i, j, \ell}(x)=2^{j d / 2} \psi_{i}\left(2^{j} x-\ell\right) .
$$

Similar to function spaces with weights we introduce sequence spaces with weights. If $w$ is a given continuous weight function, $j \in \mathbb{N}_{0}$ and $\ell \in \mathbb{Z}^{d}$ then $w(j, \ell)=w\left(2^{-j} \ell\right)$. Let $1 \leq p, q \leq \infty$. We put

$$
\begin{aligned}
& \ell_{q}\left(2^{j s} \ell_{p}\left(w_{\alpha}\right)\right):=\left\{\lambda=\left\{\lambda_{i, j, \ell}\right\}_{i, j, \ell}: \lambda_{i, j, \ell} \in \mathbb{C},\right. \\
& \left.\left\|\lambda \mid \ell_{q}\left(2^{j s} \ell_{p}(w)\right)\right\|=\left(\sum_{j=0}^{\infty} 2^{j s q}\left(\sum_{i=1}^{2^{d}-1} \sum_{\ell \in \mathbb{Z}^{d}}\left|\lambda_{i, j, \ell} w(j, \ell)\right|^{p}\right)^{q / p}\right)^{1 / q}<\infty\right\} .
\end{aligned}
$$


For smooth weights and compactly supported wavelets it makes sense to consider the Fourier-wavelet coefficients of functions $f \in L_{p}(w)$ with respect to such an orthonormal basis. The following proposition was proved in [29].

Proposition 1. Let $\phi$ be a scaling function and let $\psi_{i}, i=1, \ldots, 2^{d}-1$ be the corresponding wavelets satisfying (7). Let $1 \leq p, q \leq \infty$ and let $0<s<N_{1}$. Then a function $f \in L_{p}\left(\mathbb{R}^{d}, w_{\alpha}\right)$ belongs to $B_{p, q}^{s}\left(\mathbb{R}^{d}, w_{\alpha}\right), \alpha>0$, if and only if We put

$$
\begin{aligned}
\left\|f \mid B_{p, q}^{s}\left(\mathbb{R}^{d}, w_{\alpha}\right)\right\|^{\boldsymbol{\mu}} & =\left(\sum_{\ell \in \mathbb{Z}^{d}}\left|\left\langle f, \phi_{0, \ell}\right\rangle w_{\alpha}(\ell)\right|^{p}\right)^{1 / p} \\
& +\sum_{i=1}^{2^{d}-1}\left\{\sum_{j=0}^{\infty} 2^{j\left(s+d\left(\frac{1}{2}-\frac{1}{p}\right)\right) q}\left(\sum_{\ell \in \mathbb{Z}^{d}}\left|\left\langle f, \psi_{i, j, \ell}\right\rangle w_{\alpha}\left(2^{-j} \ell\right)\right|^{p}\right)^{q / p}\right\}^{1 / q}<\infty .
\end{aligned}
$$

Furthermore, $\left\|f \mid B_{p, q}^{s}\left(\mathbb{R}^{d}, w_{\alpha}\right)\right\|^{\text {* }}$ may be used as an equivalent norm in $B_{p, q}^{s}\left(\mathbb{R}^{d}, w_{\alpha}\right)$.

REMARK 7. There is another way of discretization of the function spaces used in this context. It is so called quarkonial decomposition. The method was developed by H. Triebel in $[52]$.

4.2. Approximation numbers of sequence spaces. Proposition 1 reduces the proof of Theorem 5 to the study of approximation numbers of the following embeddings between the sequence spaces

$$
\text { id }: \ell_{p_{0}}\left(2^{j \alpha} \ell_{p_{0}}\left(w_{\alpha}\right)\right) \rightarrow \ell_{p_{1}}\left(\ell_{p_{1}}\right)
$$

where we put

$$
\begin{aligned}
\ell_{q}\left(2^{j \alpha} \ell_{p}\left(w_{\alpha}\right)\right) & :=\left\{\lambda=\left\{\lambda_{j, \ell}\right\}_{j, \ell}: \lambda_{j, \ell} \in \mathbb{C},\right. \\
& \left.\left\|\lambda \mid \ell_{q}\left(2^{j \delta} \ell_{p}\left(w_{\alpha}\right)\right)\right\|=\left(\sum_{j=0}^{\infty} 2^{j \delta q}\left(\sum_{\ell \in \mathbb{Z}^{d}}\left|\lambda_{j, \ell} w_{j, \ell}\right|^{p}\right)^{q / p}\right)^{1 / q}<\infty\right\},
\end{aligned}
$$

where $w_{j, \ell}=w_{\alpha}\left(2^{-j} \ell\right)$. We recall that $\alpha=\delta=\left(s_{0}-\frac{d}{p_{0}}\right)-\left(s_{1}-\frac{d}{p_{1}}\right)$. The finite summation on $i=1,2, \ldots, 2^{d}-1$ is irrelevant and can be omitted.

To estimate the asymptotic behavior of the approximation numbers of the embeddings (11) we divide the source space into two parts: the local part and the global part. Namely we put

$$
\begin{aligned}
\ell_{q}\left(2^{j \alpha} \ell_{p}^{2^{j d}}\left(w_{\alpha}\right)\right) & =\left\{\lambda=\left(\lambda_{j, \ell}\right) \in \ell_{q}\left(2^{j \alpha} \ell_{p}\left(w_{\alpha}\right)\right): \lambda_{j, \ell}=0 \quad \text { if } \quad \ell>2^{j d}\right\}, \\
\ell_{q}\left(2^{j \alpha} \widetilde{\ell}_{p}\left(w_{\alpha}\right)\right) & =\left\{\lambda=\left(\lambda_{j, \text { ell }}\right) \in \ell_{q}\left(2^{j \alpha} \ell_{p}\left(w_{\alpha}\right)\right): \lambda_{j, \ell}=0 \quad \text { if } \quad \ell \leq 2^{j d}\right\} .
\end{aligned}
$$

The weight does not influence the behavior of the embeddings of the local part since then $w_{\alpha}\left(2^{-j} k\right) \sim 1$, but its influence is crucial for the second part.

The following estimates of approximation numbers of embeddings of finite dimensional sequence spaces are well known.

LEMMA 1. Let $N \in \mathbb{N}$ and $k \leq \frac{N}{4}$.

(i) If $1 \leq p_{0} \leq p_{1} \leq 2$ or $2 \leq p_{0} \leq p_{1} \leq \infty$ then

$$
a_{k}\left(\mathrm{id}: \ell_{p_{0}}^{N} \rightarrow \ell_{p_{1}}^{N}\right) \sim 1 .
$$


(ii) If $1 \leq p_{0}<2<p_{1} \leq \infty,\left(p_{0}, p_{1}\right) \neq(1, \infty)$ then

$$
a_{k}\left(\mathrm{id}: \ell_{p_{0}}^{N} \rightarrow \ell_{p_{1}}^{N}\right) \sim \min \left(1, N^{1 / t} k^{-1 / 2}\right)
$$

where $\frac{1}{t}=\frac{1}{\min \left(p_{0}^{\prime}, p_{1}\right)}$.

The above lemma is essentially due to E. D. Gluskin [15], cf. also [35] and [14].

For $p_{1}<p_{0}$ the corresponding approximation numbers are calculated by A. Pietsch, cf. $[37$, p. 109].

Lemma 2. Let $1 \leq p_{1}<p_{0} \leq \infty$. Then

$$
a_{k}\left(\text { id }: \ell_{p_{0}}^{N} \rightarrow \ell_{p_{1}}^{N}\right)=(N-k+1)^{1 / p_{1}-1 / p_{0}}, \quad k=1, \ldots, N
$$

The following extension of the estimates of the second part of Lemma 1 will be also useful, cf. [5] and [43].

Lemma 3. Let $1 \leq p_{0}<2<p_{1} \leq \infty,\left(p_{0}, p_{1}\right) \neq(1, \infty)$, and $N=1,2,3, \ldots$ Then there is a positive constant $C$ independent of $N$ and $k$ such that

$$
a_{k}\left(\mathrm{id}: \ell_{p_{0}}^{N} \rightarrow \ell_{p_{1}}^{N}\right) \leq C \begin{cases}1 & \text { if } k \leq N^{2 / t}, \\ N^{1 / t} k^{-1 / 2} & \text { if } N^{2 / t}<k \leq N, \\ 0 & \text { if } k>N,\end{cases}
$$

where $\frac{1}{t}=\max \left(\frac{1}{p_{1}}, \frac{1}{p_{0}^{\prime}}\right)$.

First we prove the estimates of the local part.

Proposition 2. Let $\alpha>0$ and let $d>0$ be a positive integer. There exist a positive constant $C>0$ independent of $k$ such that

$$
a_{k}\left(\operatorname{id}_{1}: \ell_{q_{0}}\left(2^{j \alpha} \ell_{p_{0}}^{2^{j d}}\left(w_{\alpha}\right)\right) \rightarrow \ell_{q_{1}}\left(\ell_{p_{1}}^{2^{j d}}\right)\right) \leq C k^{-\beta}
$$

where

$$
\beta= \begin{cases}\frac{\alpha}{d}+\frac{1}{2}-\frac{1}{t} & \text { if } 1 \leq p_{0}<2<p_{1} \leq \infty,\left(p_{0}, p_{1}\right) \neq(1, \infty), \text { and } \frac{\alpha}{d}>\frac{1}{t}, \\ \frac{\alpha}{d} \frac{t}{2} & \text { if } 1 \leq p_{0}<2<p_{1} \leq \infty,\left(p_{0}, p_{1}\right) \neq(1, \infty), \text { and } \frac{\alpha}{d} \leq \frac{1}{t}, \\ \frac{\alpha}{d} & \text { if } 1 \leq p_{0} \leq p_{1} \leq 2 \text { or } 2 \leq p_{0} \leq p_{1} \leq \infty, \\ \frac{\alpha}{d}+\frac{1}{p_{0}}-\frac{1}{p_{1}} & \text { if } \tilde{p}<p_{1}<p_{0} \leq \infty\end{cases}
$$

and $\frac{1}{t}=\frac{1}{\min \left\{p_{0}^{\prime}, p_{1}\right\}}, \frac{1}{\tilde{p}}=\frac{\alpha}{d}+\frac{1}{p_{0}}$.

Proof. Step 1. Preparations. Let

$$
\Lambda:=\left\{\lambda=\left(\lambda_{j, \ell}\right)_{j \in \mathbb{N}_{0}, 0 \leq \ell \leq 2^{j d}}: \lambda_{j, \ell} \in \mathbb{C}, j \in \mathbb{N}_{0}, 0 \leq \ell \leq 2^{j d}\right\} .
$$

and

$$
A_{0}=\ell_{q_{0}}\left(2^{j \delta} \ell_{p_{0}}^{2^{j d}}\right) \text { and } A_{1}=\ell_{q_{1}}\left(\ell_{p_{1}}^{2^{j d}}\right)
$$

Then

$$
a_{k}\left(\ell_{q_{0}}\left(2^{j \delta} \ell_{p_{0}}^{2^{j d}}\left(w_{\alpha}\right)\right) \rightarrow \ell_{q_{1}}\left(\ell_{p_{1}}^{2^{j d}}\right)\right) \leq C a_{k}\left(A_{0} \rightarrow A_{1}\right),
$$

since $w_{\alpha}\left(2^{-j} \ell\right) \sim 1$ if $\left.0 \leq \ell\right) \leq 2^{j d}$. Let $P_{j}: \Lambda \rightarrow \Lambda$ be the canonical projection onto $j$-level i.e. for $\lambda=\left(\lambda_{j, \ell}\right)$ we put

$$
\left(P_{j} \lambda\right)_{\ell}:=\left\{\begin{array}{ll}
\lambda_{k, \ell} & \text { if } k=j, \\
0 & \text { otherwise, }
\end{array} \quad \ell \in \mathbb{N}_{0} .\right.
$$


Then $\mathrm{id}_{1}=\sum_{j=0}^{\infty} P_{j}$. The elementary properties of the approximation numbers yield for any $j \in \mathbb{N}_{0}$

$$
a_{k}\left(P_{j}: A_{0} \rightarrow A_{1}\right) \leq 2^{-j \delta} a_{k}\left(\mathrm{id}: \ell_{p_{0}}^{2^{j d}} \rightarrow \ell_{p_{1}}^{2^{j d}}\right) .
$$

Step 2. Let $p_{0} \leq p_{1}$. The estimates of approximation numbers between finite dimensional spaces imply

$$
k^{\frac{1}{r}} a_{k}\left(\text { id }: \ell_{p_{0}}^{N} \rightarrow \ell_{p_{1}}^{N}\right) \leq C \begin{cases}N^{\frac{2}{t r}} & \text { for } k \leq N^{\frac{2}{t}}, \\ N^{\frac{1}{t}+\frac{1}{r}-\frac{1}{2}} & \text { for } \frac{1}{r}-\frac{1}{2}>0 \text { and } N^{\frac{2}{t}}<k \leq N, \\ N^{\frac{2}{t r}} & \text { for } \frac{1}{r}-\frac{1}{2} \leq 0 \text { and } N^{\frac{2}{t}}<k \leq N, \\ 0 & \text { for } k>N,\end{cases}
$$

if $1 \leq p_{0}<2<p_{1} \leq \infty, r>0$, cf. Lemma 3 , and

$$
k^{\frac{1}{r}} a_{k}\left(\mathrm{id}: \ell_{p_{0}}^{N} \rightarrow \ell_{p_{1}}^{N}\right) \leq C N^{1 / r},
$$

if $1 \leq p_{0} \leq p_{1} \leq 2$ or $2 \leq p_{0} \leq p_{1} \leq \infty$, cf. Lemma 1 .

As a consequence we get for $N=2^{j d}$

$$
L_{r, \infty}^{(a)}\left(P_{j}\right) \leq C 2^{-j \delta}\left\{\begin{array}{lr}
2^{j d \frac{2}{t r}} & \text { for } \frac{1}{r} \leq \frac{1}{2} \text { and } \\
& 1 \leq p_{0} \leq 2<p_{1} \leq \infty, \\
2^{j d\left(\frac{1}{t}+\frac{1}{r}-\frac{1}{2}\right)} r & \text { for } \frac{1}{r}>\frac{1}{2} \text { and } \\
1 & \leq p_{0}<2<p_{1} \leq \infty, \\
2^{j d \frac{1}{r}} & \text { for } 1 \leq p_{0} \leq p_{1} \leq 2 \text { or } \\
& 2 \leq p_{0} \leq p_{1} \leq \infty .
\end{array}\right.
$$

Now, for given $M \in \mathbb{N}_{0}$ let

$$
P:=\sum_{j=0}^{M} P_{j} \quad \text { and } \quad Q:=\sum_{j=M+1}^{\infty} P_{j} .
$$

The expression $L_{r, \infty}^{(a)}(T)$ is a quasi-norm of the operator ideal $\mathcal{L}_{r, \infty}^{(a)}$ therefore there exists a number $0<\varrho \leq 1$ such that

$$
L_{r, \infty}^{(a)}(P)^{\varrho} \leq C \sum_{j=0}^{M} L_{r, \infty}^{(a)}\left(P_{j}\right)^{\varrho}, \quad L_{r, \infty}^{(a)}(Q)^{\varrho} \leq C \sum_{j=M+1}^{\infty} L_{r, \infty}^{(a)}\left(P_{j}\right)^{\varrho} .
$$

Due to (19), (21), and elementary properties of geometric series we have the estimate

$$
L_{r, \infty}^{(a)}(Q)^{\varrho} \leq \begin{cases}2^{M d \varrho\left(\frac{2}{t r}-\frac{\delta}{d}\right)} & \text { for } \frac{1}{r}<\min \left\{\frac{1}{2}, \frac{\delta t}{d 2}\right\}, \\ & 1 \leq p_{0}<2<p_{1} \leq \infty, \\ \left.2^{M d \varrho\left(\frac{1}{t}+\frac{1}{r}-\frac{\delta}{d}-\frac{1}{2}\right)}\right) & \text { for } \frac{1}{2}<\frac{1}{r}<\frac{1}{2}+\frac{\delta}{d}-\frac{1}{t}, \\ & 1 \leq p_{0}<2<p_{1} \leq \infty \\ 2^{M d \varrho\left(\frac{1}{r}-\frac{\delta}{d}\right)} & \text { for } \frac{1}{r}<\frac{\delta}{d}, 1 \leq p_{0} \leq p_{1} \leq 2 \\ & \text { or } 2 \leq p_{0} \leq p_{1} \leq \infty .\end{cases}
$$

Now, if $1 \leq p_{0} \leq p_{1} \leq 2$ or $2 \leq p_{0} \leq p_{1} \leq \infty$ then (22) implies

$$
a_{2^{M d}}(Q) \leq C 2^{-M d \frac{\alpha}{d}},
$$


with the constant $C$ independent of $M$. In a similar way if $1 \leq p_{0}<2<p_{1} \leq \infty$ then

$$
a_{k}(Q) \leq C \begin{cases}k^{-\left(\frac{\alpha}{d}+\frac{1}{2}-\frac{1}{t}\right)} & \text { if } \frac{\alpha}{d}>\frac{1}{t}, \\ k^{-\frac{\alpha}{d} \frac{t}{2}} & \text { if } \frac{\alpha}{d} \leq \frac{1}{t}\end{cases}
$$

with $k=2^{M d}$ if $\frac{\alpha}{d}>\frac{1}{t}$ and $k=\left[2^{M d 2 / t}\right]$ otherwise. Here $[x]$ denotes the integer part of $x$. By monotonicity of approximation numbers we extend the inequalities (23) and (24) to any positive integer $k$.

The estimate of the approximation numbers of the operator $P$ goes in a similar way. The only difference is that we should choose a different value of $r$ to sum up the corresponding sums. Namely we have

$$
L_{r, \infty}^{(a)}(P)^{\varrho} \leq C\left\{\begin{array}{lr}
2^{M d \varrho\left(\frac{2}{t r}-\frac{\delta}{d}\right)} & \text { for } \frac{\delta t}{d 2}<\frac{1}{r}<\frac{1}{2}, \\
& 1 \leq p_{0}<2<p_{1} \leq \infty, \\
\left.2^{M d \varrho\left(\frac{1}{t}+\frac{1}{r}-\frac{\delta}{d}-\frac{1}{2}\right)}\right) & \text { for } \frac{1}{r}>\max \left\{\frac{1}{2}, \frac{1}{2}+\frac{\delta}{d}-\frac{1}{t}\right\}, \\
& 1 \leq p_{0} \leq 2<p_{1} \leq \infty, \\
2^{M d \varrho\left(\frac{1}{r}-\frac{\delta}{d}\right)} & \text { for } \frac{1}{r}>\frac{\delta}{d}, 1 \leq p_{0} \leq p_{1} \leq 2 \\
& \text { or } 2 \leq p_{0}<p_{1} \leq \infty .
\end{array}\right.
$$

Now, if $1 \leq p_{0} \leq p_{1} \leq 2$ or $2 \leq p_{0} \leq p_{1} \leq \infty$ then (25) implies

$$
a_{2^{M d}}(P) \leq C 2^{-M d \frac{\alpha}{d}} .
$$

In a similar way if $1 \leq p_{0}<2<p_{1} \leq \infty$ then

$$
a_{k}(P) \leq C \begin{cases}k^{-\left(\frac{\alpha}{d}+\frac{1}{2}-\frac{1}{t}\right)} & \text { if } \frac{\alpha}{d} \geq \frac{1}{t}, \\ k^{-\frac{\alpha}{d} \frac{t}{2}} & \text { if } \frac{\alpha}{d}<\frac{1}{t} .\end{cases}
$$

with $k=2^{M d}$ if $\frac{\alpha}{d}>\frac{1}{t}$ and $k=\left[2^{M d 2 / t}\right]$ otherwise.

Step 3. Let $\tilde{p}<p_{1}<p_{0}$. Now using the Pietsch estimates, cf. Lemma 2, we get

$$
k^{\frac{1}{r}} a_{k}\left(\mathrm{id}: \ell_{p_{0}}^{N} \rightarrow \ell_{p_{1}}^{N}\right) \leq C N^{\frac{1}{r}+\frac{1}{p_{1}}-\frac{1}{p_{0}}} .
$$

In consequence

$$
L_{r, \infty}^{(a)}\left(P_{j}\right) \leq C 2^{-j \delta} 2^{j d\left(\frac{1}{r}+\frac{1}{p_{1}}-\frac{1}{p_{0}}\right)} .
$$

Summing up as in the previous step we get

$$
L_{r, \infty}^{(a)}(Q) \leq C 2^{M d\left(\frac{1}{r}+\frac{1}{p_{1}}-\frac{1}{p_{0}}-\frac{\alpha}{d}\right)}
$$

if $0<\frac{1}{r}<\frac{\alpha}{d}+\frac{1}{p_{0}}-\frac{1}{p_{1}}$. So,

$$
a_{2^{M d}}(Q) \leq C 2^{-M d\left(\frac{\alpha}{d}+\frac{1}{p_{0}}-\frac{1}{p_{1}}\right)}
$$

In an analogous way

$$
L_{r, \infty}^{(a)}(P) \leq C 2^{M d\left(\frac{1}{r}+\frac{1}{p_{1}}-\frac{1}{p_{0}}-\frac{\alpha}{d}\right)}
$$

if $\frac{1}{r}>\frac{\alpha}{d}+\frac{1}{p_{0}}-\frac{1}{p_{1}}$ and

$$
a_{2^{M d}}(P) \leq C 2^{-M d\left(\frac{\alpha}{d}+\frac{1}{p_{0}}-\frac{1}{p_{1}}\right)}
$$

This finishes the proof. 
It remains to consider the global part. This is done in the next proposition.

Proposition 3. Let $\alpha>0$ and let $d>0$ be a positive integer. There exists a positive constant $C>0$ independent of $k$ such that

$$
a_{k}\left(\operatorname{id}_{2}: \ell_{p_{0}}\left(2^{j \alpha} \tilde{\ell}_{p_{0}}\left(w_{\alpha}\right)\right) \rightarrow \ell_{p_{1}}\left(\tilde{\ell}_{p_{1}}\right)\right) \sim k^{-\beta}(1+\log k)^{\alpha / d},
$$

where

$$
\beta= \begin{cases}\frac{\alpha}{d}+\frac{1}{2}-\frac{1}{t} & \text { if } 1 \leq p_{0}<2<p_{1} \leq \infty,\left(p_{0}, p_{1}\right) \neq(1, \infty), \text { and } \frac{\alpha}{d}>\frac{1}{t}, \\ \frac{\alpha}{d} \frac{t}{2} & \text { if } 1 \leq p_{0}<2<p_{1} \leq \infty,\left(p_{0}, p_{1}\right) \neq(1, \infty), \text { and } \frac{\alpha}{d} \leq \frac{1}{t}, \\ \frac{\alpha}{d} & \text { if } 1 \leq p_{0} \leq p_{1} \leq 2 \text { or } 2 \leq p_{0} \leq p_{1} \leq \infty, \\ \frac{\alpha}{d}+\frac{1}{p_{0}}-\frac{1}{p_{1}} & \text { if } \tilde{p}<p_{1}<p_{0} \leq \infty\end{cases}
$$

and $\frac{1}{t}=\frac{1}{\min \left\{p_{0}^{\prime}, p_{1}\right\}}, \frac{1}{\tilde{p}}=\frac{\alpha}{d}+\frac{1}{p_{0}}$.

Proof. Step 1. Preparations. If $|\ell|>2^{j}, \ell \in \mathbb{Z}^{d}$, then $\left(1+\left|2^{-j} \ell\right|^{2}\right)^{\alpha / 2} \sim 2^{-j \alpha}|\ell|^{\alpha}$. So,

$$
a_{k}\left(\operatorname{id}_{2}\right) \sim a_{k}\left(\ell_{p_{0}}\left(\tilde{\ell}_{p_{0}}\left(v_{\alpha}\right)\right) \rightarrow \ell_{p_{1}}\left(\tilde{\ell}_{p_{1}}\right)\right),
$$

where $v_{\alpha}(j, \ell)=\ell^{\alpha / d}$. The norm in the space $\ell_{p}\left(\tilde{\ell}_{p}\left(v_{\alpha}\right)\right)$ is given by

$$
\left\|\lambda \mid \ell_{p}\left(\tilde{\ell}_{p}\left(v_{\alpha}\right)\right)\right\|=\left(\sum_{j=0}^{\infty} \sum_{\ell=2^{j d}+1}^{\infty}\left|\lambda_{j, \ell} \ell^{\alpha / d}\right|^{p}\right)^{1 / p} .
$$

If $2^{n d} \leq \ell<2^{(n+1) d}$ then $\ell^{\alpha / d} \sim 2^{n \alpha}$. So the condition

$$
2^{n d} \leq \ell<2^{(n+1) d} \quad \text { and } \quad \lambda_{j, \ell} \neq 0
$$

can be satisfied on any $j$-level with $j \leq n$ for at most $2^{j d}\left(2^{d}-1\right)$ entries. In consequence taking $N_{j}=j 2^{j d}\left(2^{d}-1\right)$ and changing the order of summation in (35) we get

$$
\left\|\lambda\left|\ell_{p}\left(\tilde{\ell}_{p, w_{\alpha}}\right)\|\sim\| \lambda\right| \ell_{p}\left(2^{j \alpha} \ell_{p}^{N_{j}}\right)\right\|
$$

So it follows from $(34)-(36)$ that

$$
a_{k}\left(\operatorname{id}_{2}\right) \sim a_{k}\left(\operatorname{id}_{3}: \ell_{p_{0}}\left(2^{j \alpha} \ell_{p_{0}}^{N_{j}}\right) \rightarrow \ell_{p_{1}}\left(\ell_{p_{1}}^{N_{j}}\right)\right) .
$$

Now to prove the estimates from above we can deal as in the proof of Proposition 2. So we only sketch the argument. Let the operators $P_{j}, P$ and $Q$ be the same as above. We have

$$
a_{k}\left(P_{j}\right) \leq 2^{-j \alpha} a_{k}\left(\text { id }: \ell_{p_{0}}^{N_{j}} \rightarrow \ell_{p_{1}}^{N_{j}}\right),
$$

Step 2. We prove the estimates from above. Let $p_{0} \leq p_{1}$. Using (17), (18) and (28) we get

$$
L_{r, \infty}^{(a)}\left(P_{j}\right) \leq C 2^{-j \alpha} \begin{cases}N_{j}^{\frac{2}{t r}} & \text { for } \frac{1}{r} \leq \frac{1}{2} \text { and } \\ & 1 \leq p_{0}<2<p_{1} \leq \infty, \\ N_{j}^{\frac{1}{t}+\frac{1}{r}-\frac{1}{2}} & \text { for } \frac{1}{r}>\frac{1}{2} \text { and } \\ & 1 \leq p_{0}<2<p_{1} \leq \infty, \\ N_{j}^{\frac{1}{r}} & \text { for } 1 \leq p_{0} \leq p_{1} \leq 2 \text { or } 2 \leq p_{0} \leq p_{1} \leq \infty, \\ N_{j}^{\frac{1}{r}+\frac{1}{p_{1}}-\frac{1}{p_{0}}} & \text { for } \tilde{p}<p_{1}<p_{0} \leq \infty .\end{cases}
$$


Now, for chosen $M \in \mathbb{N}_{0}$ the formulae (20) and (39) imply

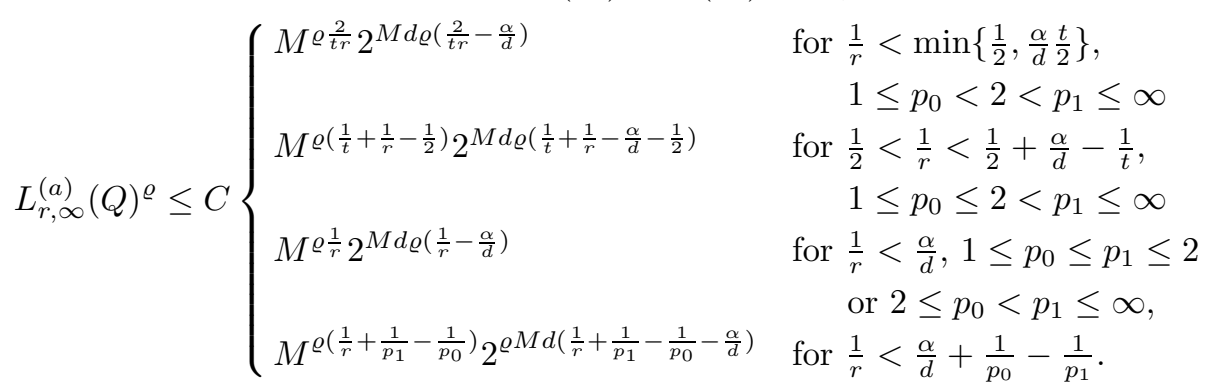

If $1 \leq p_{0} \leq p_{1} \leq 2$ or $2 \leq p_{0} \leq p_{1} \leq \infty$ then (40) implies

$$
a_{M 2^{M d}}(Q) \leq C 2^{-M d \frac{\alpha}{d}} \leq C\left(1+\log \left(M 2^{M d}\right)\right)^{\frac{\alpha}{d}}\left(M 2^{M d}\right)^{-\frac{\alpha}{d}} .
$$

In a similar way if $1 \leq p_{0}<2<p_{1} \leq \infty$ then

$$
a_{k}(Q) \leq C \begin{cases}(1+\log (k))^{\frac{\alpha}{d}} k^{-\left(\frac{\alpha}{d}+\frac{1}{2}-\frac{1}{t}\right)} & \text { if } \frac{\alpha}{d}>\frac{1}{t}, \\ (1+\log (k))^{\frac{\alpha}{d}} k^{-\frac{\alpha}{d} \frac{t}{2}} & \text { if } \frac{\alpha}{d} \leq \frac{1}{t} .\end{cases}
$$

with $k=M 2^{M d}$ if $\frac{\alpha}{d}>\frac{1}{t}$ and $k=\left[\left(M 2^{M d}\right)^{2 / t}\right]$ otherwise.

Finally, if $\tilde{p}<p_{1}<p_{0}$ then

$$
a_{M 2^{M d}}(Q) \leq C\left(1+\log \left(M 2^{d M}\right)\right)^{\frac{\alpha}{d}}\left(M 2^{M d}\right)^{-\left(\frac{\alpha}{d}+\frac{1}{p_{0}}-\frac{1}{p_{1}}\right)} .
$$

The proof of the estimates for the operator $P$ goes in a similar way. This proves the estimates from above.

Step 3. It remains to consider the estimates from below. We consider the following commutative diagram

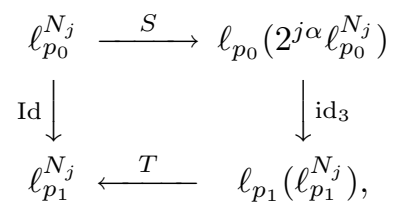

where

$$
\begin{aligned}
(S(\nu))_{i, \ell} & = \begin{cases}\nu_{\ell} & \text { if }(i, \ell)=(j, \ell), \\
0 & \text { otherwise }\end{cases} \\
(T(\lambda))_{\ell} & =\lambda_{j, \ell}, \quad 1 \leq \ell \leq N_{j} .
\end{aligned}
$$

Then

$$
a_{k}(\mathrm{Id}) \leq\|S\| a_{k}\left(\mathrm{id}_{3}\right) .
$$

Taking $k=j 2^{d j-2}$ we get by (37) and Lemma 1 or Lemma 2

$$
\begin{aligned}
& 1 \leq C a_{k}(\mathrm{Id}) \leq C 2^{j \alpha} a_{k}\left(\mathrm{id}_{2}\right) \leq C\left(k(1+\log k)^{-1}\right)^{\alpha / d} a_{k}\left(\mathrm{id}_{2}\right), \\
& \text { if } 1 \leq p_{0} \leq p_{1} \leq 2 \text { or } 2 \leq p_{0} \leq p_{1} \leq \infty, \\
& \left(j 2^{d j-2}\right)^{\frac{1}{t}-\frac{1}{2}} \leq C a_{k}(\mathrm{Id}) \leq C 2^{j \alpha} a_{k}\left(\mathrm{id}_{2}\right), \\
& \text { if } 1 \leq p_{0}<2<p_{1} \leq \infty,\left(p_{0}, p_{1}\right) \neq(1, \infty) \text { and } \frac{\alpha}{d}>\frac{1}{t}, \\
& \left(j 2^{d j}\right)^{\frac{1}{p_{1}}-\frac{1}{p_{0}}} \leq C a_{k}(\mathrm{Id}) \leq C 2^{j \alpha} a_{k}\left(\mathrm{id}_{2}\right), \quad \text { if } \quad \tilde{p}<p_{1}<p_{0} \leq \infty .
\end{aligned}
$$


For $1 \leq p_{0}<2<p_{1} \leq \infty,\left(p_{0}, p_{1}\right) \neq(1, \infty)$ and $\frac{\alpha}{d} \leq \frac{1}{t}$ we take $k=\left[\left(j 2^{j d}\right)^{2 / t}\right]$. Then

$$
1 \leq C a_{k}(\mathrm{Id}) \leq C 2^{j \alpha} a_{k}\left(\mathrm{id}_{2}\right) .
$$

This finishes the proof.

Since id $=\mathrm{id}_{1}+\mathrm{id}_{2}$, cf. (11)-(13), it follows from Proposition 2 and Proposition 3 that

$$
a_{k}(\mathrm{id}) \sim k^{-\beta}(1+\log k)^{\alpha / d}
$$

where $\beta$ takes the same values as in Proposition 3. Now Theorem 5 follows from the above estimate and Proposition 1.

\section{References}

[1] D. Eh. Allakhverdiev, On the rate of approximation of completely continuous operators by finite dimensional operators, Azerbajzhan Gos. Univ. Uchen. Zap. 2 (1957), 27-37.

[2] G. Bourdaud, Ondelettes et espaces de Besov, Revista Math. Iberoam. 11 (1995), 477-512.

[3] M. S. Birman, M. Z. Solomyak, Piecewise polynomial approximations of functions of the class $W_{p}^{\alpha}$, Mat. Sb. 73 (1967), 331-355.

[4] H. Brézis and S. Wainger, A note of limiting cases of Sobolev embeddings and convolution inequalities, Comm. Partial Differential Equations 5 (1980), 773-789.

[5] A. M. Caetano, About approximation numbers in function spaces, J. Approx. Theory 94 (1998), 383-395.

[6] B. Carl, Entropy numbers, s-numbers and eigenvalue problems, J. Func. Anal. 41 (1981), 290-306.

[7] B. Carl and I. Stephani, Entropy, Compactness and the Approximation of Operators, Cambridge Univ. Press, Cambridge, 1990.

[8] B. Carl and H. Triebel, Inequalities between eigenvalues, entropy numbers, and related quantities of compact operators in Banach spaces, Math. Ann. 251 (1980), 129-133.

[9] F. Cobos and T. Kühn, Entropy numbers of embedings of Besov spaces in generalized Lipschitz spaces, J. Approx. Theory 112 (2001), 73-92.

[10] S. Coleman, V. Glazer and A. Martin, Action minima among solutions to a class of Euclidean scalar field equations, Comm. Math. Phys. 58 (1978), 211-221.

[11] D. E. Edmunds and D. D. Haroske, Embeddings in spaces of Lipschitz type, entropy and approximation numbers, and applications, J. Approx. Theory 104 (2000), 226-271.

[12] D. E. Edmunds and H. Triebel, Entropy numbers and approximation numbers in function spaces, Proc. London Math. Soc. 58 (1989), 137-152.

[13] D. E. Edmunds and H. Triebel, Entropy numbers and approximation numbers in function spaces II, Proc. London Math. Soc. 64 (1992), 153-169.

[14] D. E. Edmunds and H. Triebel, Function Spaces, Entropy Numbers, Differential Operators, Cambridge Univ. Press, Cambridge, 1996.

[15] E. D. Gluskin, Norms of random matrices and diameter of finite dimensional sets, Mat. Sb. 120 (1983), 180-189 (in Russian).

[16] D. Haroske, Approximation numbers in some weighted function spaces, Journal of Approx. Theory 83 (1995), 104-136.

[17] D. Haroske, Embeddings of some weighted function spaces on $\mathbb{R}^{n}$; entropy and approximation numbers, An. Univ. Craiova Ser. Mat. Inform. 24 (1997), 1-44. 
[18] D. Haroske, Some limiting embeddings of in weighted function spaces and related entropy numbers, Jenaer Schriften zur Mathematik und Informatik Math/Inf/97/04, 1997, 54 pp.

[19] D. Haroske and H. Triebel, Entropy numbers in weighted function spaces and eigenvalue distributions of some degenerate pseudo differential operators I, Math. Nachr. 167 (1994), 131-156.

[20] D. Haroske and H. Triebel, Entropy numbers in weighted function spaces and eigenvalue distributions of some degenerate pseudo differential operators II, Math. Nachr. 168 (1994), 109-137.

[21] D. Haroske and H. Triebel, Wavelet bases and entropy numbers in weighted function spaces, Math. Nachr. 278 (2005), 108-132

[22] A. N. Kolmogorov, Über die beste Annäherung von Funktionen einer gegebenen Funktionenklasse, Ann. Math. 37 (1936), 107-110.

[23] A. N. Kolmogorov and V. M. Tikhomirov, $\varepsilon$-entropy and $\varepsilon$-capacity of sets in function spaces, Uspekhi Mat. Nauk 14 (1959), 3-86 (in Russian).

[24] V. I. Kondrashov, Certain properties of functions in the spaces $L^{p}$, Dokl. Akad. Nauk SSSR 48 (1945), 563-566 (in Russian).

[25] H. König, Eigenvalue Distribution of Compact Operators, Birkhäuser, Basel, 1986.

[26] T. Kühn, Compact embeddings of Besov spaces in exponential Orlicz spaces, J. London Math. Soc. 67 (2003), 235-244.

[27] Th. Kühn, H.-G. Leopold, W. Sickel and L. Skrzypczak, Entropy numbers of Sobolev embeddings of radial Besov spaces, J. Approx. Theory 121 (2003), 244-268.

[28] Th. Kühn, H.-G. Leopold, W. Sickel and L. Skrzypczak, Entropy numbers of embeddings of weighted Besov spaces, Jenaer Schriften zur Mathematik und Informatik Math/Inf/13/03, 2003, 57 pp.

[29] Th. Kühn, H.-G. Leopold, W. Sickel and L. Skrzypczak, Entropy numbers of embeddings of weighted Besov spaces, Constr. Approx. 23 (2006), 61-77.

[30] Th. Kühn, H.-G. Leopold, W. Sickel and L. Skrzypczak, Entropy numbers of embeddings of weighted Besov spaces. II, Proc. Roy. Soc. Edinburgh Sect. A (to appear).

[31] Th. Kühn, H.-G. Leopold, W. Sickel and L. Skrzypczak, Entropy numbers of embeddings of weighted Besov spaces III: weights of logarithmic type, Math. Z., to appear.

[32] P. L. Lions, Symmétrie et compacité dans les espaces de Sobolev, J. Funct. Anal. 49 (1982), 315-334.

[33] J. Löfström, Interpolation of weighted spaces of differentiable functions on $\mathbb{R}^{d}$, Ann. Mat. Pura Appl. 132 (1982), 189-214.

[34] Y. Meyer, Wavelets and Operators, Cambridge Univ. Press, Cambridge, 1992.

[35] K. Mynbaev and M. Otel'baev, Weighted Functional Spaces and Differential Operators Spectrum, Nauka, Moscow, 1988 (in Russian).

[36] A. Pietsch, Operator Ideals, North-Holland, Amsterdam, 1980.

[37] A. Pietsch, Eigenvalues and s-numbers, Akad. Verlagsges. Geest \& Portig, Leipzig, 1987.

[38] L. S. Pontryagin and L. G. Schnirelmann, Sur une propriété métrique de la dimension, Ann. Math. 33 (1932), 152-62.

[39] S. Roudenko, Matrix-weighted Besov spaces, Trans. Amer. Math. Soc., 355 (2002), 273314.

[40] V. S. Rychkov, Littlewood-Paley theory and function spaces with $A_{p}^{\ell o c}$ weights, Math. Nachr. 224 (2001), 145-180.

[41] H.-J. Schmeißer and H. Triebel, Topics in Fourier Analysis and Function Spaces, Akad. Verlagsgesellschaft Geest \& Portig, Leipzig, 1987. 
[42] L. Skrzypczak, Entropy numbers of Trudinger-Strichartz embeddings of radial Besov spaces and applications, J. London Math. Soc. 53 (2004), 569-581.

[43] L. Skrzypczak, On approximation numbers of Sobolev embedings of weighted function spaces, J. Approx. Theory 136 (2005), 91-107.

[44] L. Skrzypczak and B. Tomasz, Approximation numbers of Sobolev embeddings of spaces of radial functions, Comment. Math. Tomus Specialis in honorem Iuliani Musielak (2004), 238-255.

[45] L. Skrzypczak and B. Tomasz, Entropy of Sobolev embeddings of radial functions and radial eigenvalues of Schrödinger operators on isotropic manifolds, Math. Nach. (to appear).

[46] W. A. Strauss, Existence of solitary waves in higher dimensions, Comm. in Math. Physics 55 (1977), 149-162.

[47] R. S. Strichartz, A note on Trudinger's extension of Sobolev's inequatlity, Indiana Univ. Math. J. 58 (1972), 841-842.

[48] H. Triebel, Approximation numbers and entropy numbers of embeddings of fractional Besov-Sobolev spaces in Orlicz spaces, Proc. London Math. Soc. 66 (1993), 589-618.

[49] H. Triebel, Interpolation Theory, Function Spaces, Differential Operators, North-Holland, Amsterdam, 1978.

[50] H. Triebel, Relations between approximation numbers and entropy numbers, J. Approx. Theory 78 (1994), 112-116.

[51] H. Triebel, Theory of Function Spaces, Birkhäuser, Basel, 1983.

[52] H. Triebel, The Structure of Functions, Birkhäuser, Basel, 2001.

[53] N. Trudinger, On imbeddings into Orlicz spaces and some applications, J. Math. Mech. 17 (1967), 473-483.

[54] P. Wojtaszczyk, A Mathematical Introduction to Wavelets, Cambridge Univ. Press, Cambridge, 1997. 